\title{
Spatial Interference Reduction by Subarray Stacking in Large Two-dimensional Antenna Arrays
}

\author{
Muhammad Yasir Javed, Nuutti Tervo Student Member, IEEE, Marko E. Leinonen Student Member, IEEE and \\ Aarno Pärssinen Senior Member, IEEE
}

\begin{abstract}
This paper proposes a systematic approach for stacking uniform linear arrays (ULAs) of the different sizes to reduce sidelobes and form nulls to the radiated beam pattern. In sidelobe reduction, the nulls and sidelobes of the vertically stacked ULAs are aligned to reduce the overall sidelobe level. In nulling, the nulls of varying length ULAs are aligned to configure the null direction and simultaneously reduce the sidelobes. Both approaches are relaxed to ensure the feasibility of the implementation. The stacking methods are studied by an extensive set of simulations together with azimuth and elevation beamforming. Sidelobe reduction method showed potential for better than $30 \mathrm{~dB}$ sidelobe level (SLL) and more than $20 \mathrm{~dB}$ relative null depth with less than 100 antenna elements. Finally, the concepts are validated by beam pattern measurements of a $28 \mathrm{GHz}$ 64-element, 16-chain phased array transceiver using a $100 \mathrm{MHz}$ wide 5G waveform.
\end{abstract}

Index Terms-Antenna selection, beam synthesis, interbeam interference, $\mathbf{m m W}$, sidelobe reduction, phased arrays, $5 \mathrm{G}$.

\section{INTRODUCTION}

Fifth-generation $(5 \mathrm{G})$ cellular systems are envisioned to deliver order-of-magnitude improvements over the current networks in peak data rate, network capacity, latency, and reliability [1]. Millimeter-wave ( $\mathrm{mmW}$ ) and massive multipleinput multiple-output (MIMO) are the two key physical layer technologies that enable future networks to be energy-efficient, secure, robust, and will make effective use of the available broadband spectrum [2], [3]. On the other hand, $\mathrm{mmW}$ signals are susceptible to high path loss, penetration loss, severe atmospheric absorption, and high rainfall attenuation [4]. However, antenna size and inter-element spacing in $\mathrm{mmW}$ systems are in the order of millimeters, which makes it possible to pack tens or even hundreds of elements in a compact form factor to compensate for the path loss and to provide spatial filtering for interference reduction in the far-field of the antenna array.

Large $\mathrm{mmW}$ antenna arrays use radio frequency (RF) beamforming for steering the beams in different directions. For serving multiple users simultaneously, many existing $\mathrm{mmW}$ systems implemented in practice divide the antenna panel further into subarrays [5]. Such a system is depicted in Fig. 1. Each subarray is connected to a single baseband (BB) chain

Manuscript received January 15, 2020; revised November 06, 2020; accepted November 16, 2020. This research has been financially supported by Nokia Corporation Ltd, Academy of Finland 6Genesis Flagship (grant 318927) and Business Finland project 5GForce. Keysight Technologies is acknowledged for laboratory equipment.

The authors are with the Centre for Wireless Communications Radio Technologies, University of Oulu, FI-90014 Oulu, Finland (email: yasir.javed@oulu.fi; nuutti.tervo@oulu.fi; marko.e.leinonen@oulu.fi; aarno.parssinen@oulu.fi).

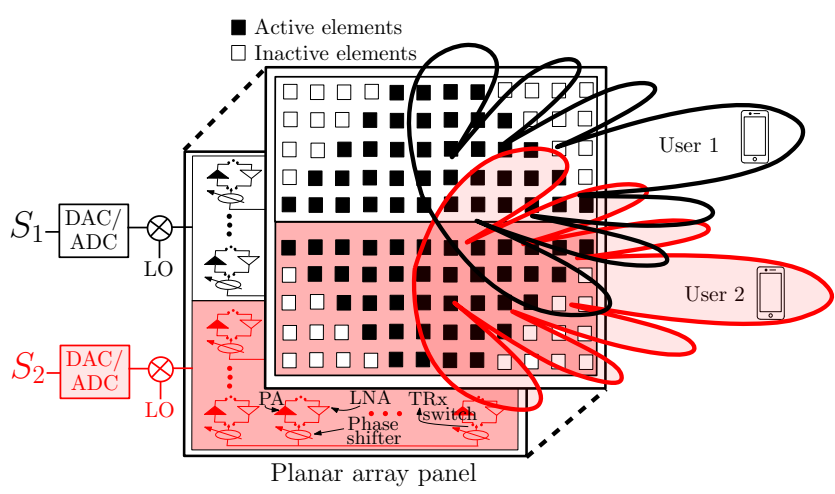

Fig. 1. Overview of the mmW antenna array system serving two users.

(i.e. digital-to-analogue(DAC) / analogue-to-digital (ADC) converter) serving a dedicated user in a dedicated direction [6]. Subarrays are usually equipped with antenna-specific RF phase shifters, which are used to steer the beams into the desired directions. However, regular and discrete array shapes such as uniform linear array (ULA) or uniform rectangular array (URA) suffer from high sidelobes when uniform amplitude excitation [7] is used. In a system where multiple beams carry different information content to serve different users, sidelobes of the phased array are potentially interference to the other main beams. The required isolation between the beams depends on the target signal-to-interference-plus-noiseratio (SINR), which again depends on the used modulation and coding scheme (MCS). For high-order modulations such as 256-quadrature amplitude modulation (QAM), more than 30 $\mathrm{dB}$ of inter-beam isolation is required to achieve decent SINR for error-free data transmission [8]. The requirement may be even higher when the users have different link distances and hence require different transmit power.

Different digital beamforming techniques such as zeroforcing (ZF) [9] or minimum mean square estimation (MMSE) [10] can be used to suppress the interference between the beams. However, these techniques rely on accurate channel state information (CSI) and antenna calibration (including inaccuracies of the phase shifters and other RF components), which is not often realistic in practice at least without the significant signalling overhead. Hence, any error in the CSI, including the inaccuracies in RF beamforming, has a significant impact on the performance when processing the signals between the beams [11], [12].

The beams can also be configured for lower interference by controlling the two-dimensional shape of the array by 
selecting the desired antenna elements for transmission and reception. Such techniques can be enabled in practice, for example, by using transceiver (TRx) switches to configure the transmit/receive mode of individual elements. However, many of such antenna selection techniques [13]-[15] existing in the literature rely on holistic optimization approach without revealing the fundamentals of the techniques that are often crucial for practical implementation. Subarray-based techniques [16]-[18] have been proposed previously, but these techniques were developed primarily for radar and satellite communication systems. Implementation of these techniques require time delays or non-uniform inter-element spacing, which is very challenging due to their larger size and higher cost for mmW circuits targeting especially for cellular systems. The method used in this paper takes into account the limits of implementation at $\mathrm{mmW}$ circuits and can be used for radar and satellite systems as well. In [19], the authors give the basic principle of how the antenna selection techniques can be systematically used for sidelobe reduction. In this paper, the basic principles of [19] are expanded for nulling and interference reduction over multiple beams. The technique configures a two-dimensional array panel for the beam shaping by utilizing the equation of the null directions.

The rest of the paper is organized as follows. Section II discusses on different spatial interference reduction techniques in phased array systems. Section III provides a comprehensive analysis of the proposed method of reducing interference. Demonstration and measurements are shown in section IV and concluded in section $\mathrm{V}$.

\section{INTERFERENCE REDUCTION IN MMW PHASED ARRAYS}

In general, the interference reduction techniques can be divided into two main categories based on whether the direction of interference is known or unknown. In this section, a short review is given for these two scenarios before presenting our approach in section III.

\section{A. Unknown Direction of Interference}

If the direction of interference is not known, the target is to reduce the maximum potential interference that is often caused by the beam pattern sidelobes. Traditional sidelobe reduction techniques discussed in [20]-[23] require amplitude control per antenna element to window the power radiated by antenna array. Windowing softens the edge effect, which is the main reason for the sidelobes. However, windowing increases the beamwidth [7] and ultra-low sidelobes requires precise control of the power applied to each antenna element. Moreover, the amplitude control deteriorates the power amplifier (PA) efficiency and limits the achievable effective isotropic radiated power (EIRP) in the [24] transmitter (Tx) while it also affects to the overall noise figure of the receiver $(\mathrm{Rx})$ [25].

Sidelobes can be also reduced by using non-identical spacing between elements, e.g. array thinning [26]-[28] and space tapering [29]. However, the drawback of these methods is that typically the spacing between the antenna elements is fixed, and the standard half of a wavelength is generally chosen to balance between antenna coupling, array gain, and grating lobes [30]. Thinned arrays obtain a spatial taper to reduce the sidelobes by turning elements off in a uniform array with a periodic lattice [31]. However, very often the inactive elements are allowed to be placed in any physical position which results in inefficient use of the antenna aperture. That is because the inactive elements cannot be allocated to other beams due to the design complexity of the $\mathrm{mmW}$ analogue beamforming systems, which are usually implemented as tiled phased arrays with rectangular shape of antennas constructed from tree-like, active feeding networks with amplitude and phase control as in [32]-[34]. That applies for example to $802.11 \mathrm{ad}$ and $5 \mathrm{G}$ cellular mmW solutions. On the other hand, placing the inactive elements to the edge of the array allows to allocate them more easily for other beams with decent design complexity of the configurable feed network. That can be implemented by multiplexing the active front-ends connected to antenna elements and allocate resources to different beams dynamically [33]. In the interleaved arrays, groups of elements dedicated to different frequencies or functions within the same aperture are interleaved, interlaced, or interspersed [35], [36] for lower sidelobes. However, due to the complexity of the feeding network, interleaving elements dedicated to multiple independent signals at $\mathrm{mmW}$ circuits are difficult to implement. Large planar arrays can also be partitioned into aperiodic two-dimensional tiles to improve the level of peak sidelobe, directivity, and efficiency of aperture [37]-[41]. These methods can, however, be seen as alternative ways of achieving array thinning. It is challenging to avoid the grating lobes in electrical beam steering with aperiodic geometries, and the range of scanning is often limited.

\section{B. Known Direction of Interference}

Zero-forcing (ZF) [9] can be used to produce nulls in the desired direction by altering the amplitude and phase excitation over the antennas. Again, changing the excitation amplitude over antenna elements from unitary limits the available EIRP similarly as in case of amplitude tapering, because the maximum power per RF branch is always limited by the maximum output power of an individual PA, implemented either on-chip or as an external component. The interference can also be cancelled over the subarray inputs/outputs by using different digital or analog cancellation techniques. Interbeam Interference Cancellation (IBIC) [42] is one of these methods to cancel beams over subarray inputs/outputs and hence over several directions. Precise cancellation by any of the digital (or analog) methods, however, requires precise amplitude and phase control because cancellation is very sensitive to errors. Accurate digital nulling requires complexvalued CSI measured through the subarrays from each user or the arrays are required to be calibrated with reasonable accuracy. Other nulling methods change the element spacing from the traditional half of the wavelength to influence null locations of the beam pattern. By controlling the spacing of each antenna element, array thinning [26], [43] or space tapering [27] can be used to produce nulls in the direction of interference. As mentioned before, these techniques are considered to be impractical for cellular communications since they require control of antenna spacing. 


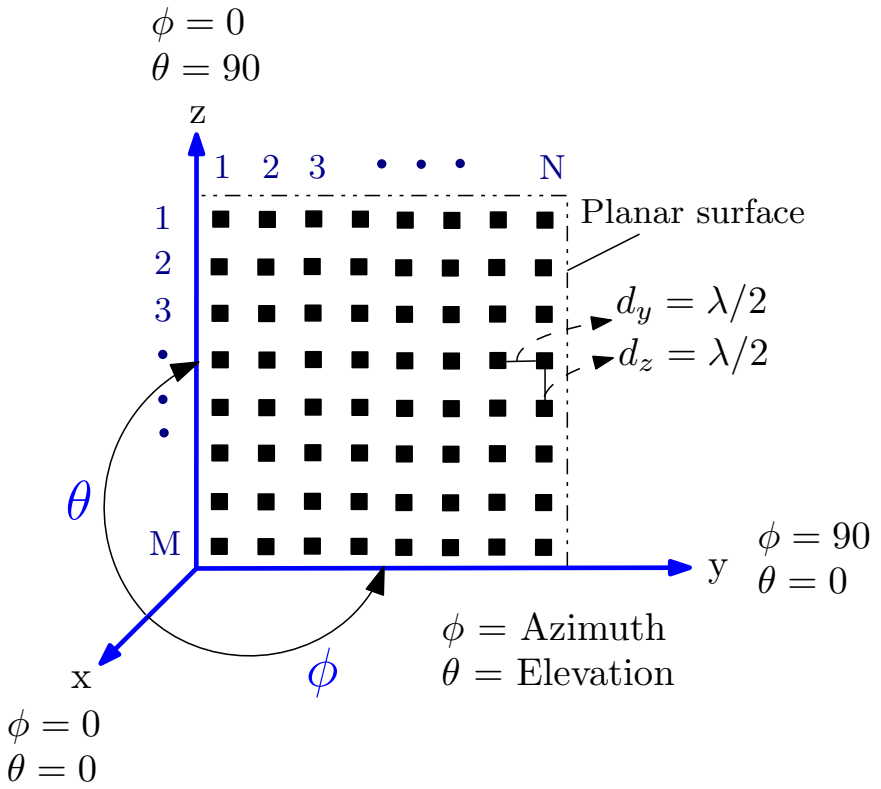

Fig. 2. Two-dimensional antenna panel with vertically stacked ULAs.

\section{Interference Reduction by SubarRay StaCking}

Fig. 2 shows the planar antenna array located in a threedimensional (3D) coordinate system. In the figure, $x>0$ is the direction of propagation (normal of the array). The azimuth angle $\phi$ is defined as the angle from positive $\mathrm{x}$-axis towards to $y$-axis, and the elevation angle $\theta$ is the angle from positive $\mathrm{x}$-axis towards the $\mathrm{z}$-axis. Consider a planar array of $\mathrm{M}$ rows and $\mathrm{N}$ columns of elements arranged along a rectangular lattice with element spacing of $d_{y}$ in the y-axis and $d_{z}$ in the z-axis. Let's further assume that the elements have uniform amplitude excitation, meaning that each element can be either on or off. The planar antenna grid is divided into rows, i.e. $N_{M}$-element uniform linear subarrays (ULSA) stacked along the z-axis. Location of nulls $\left(\phi_{\text {nulls }}\right)$ of ULSA over the azimuth plane can be calculated as [7]

$$
\phi_{\text {nulls }}=\arcsin \left[\frac{\lambda}{2 \pi d_{y}}\left(-\beta_{m y} \pm \frac{2 n_{i} \pi}{N_{M}}\right)\right],
$$

where $n_{i}=1,2,3, \ldots, n_{i} \neq N_{M}, 2 N_{M}, 3 N_{M}, \ldots$ is the null index. $\lambda$ is wavelength and $\beta_{m y}=-2 \pi d_{y} \sin \left(\phi_{s}\right) / \lambda$ is the phase progression of $m^{\text {th }}$ subarray for steering beam in azimuth direction $\phi_{s}$.

\section{A. Subarray Stacking for Sidelobe Reduction}

The sidelobes over a certain azimuth cut can be reduced by stacking multiple rows of different sizes on top of each other. By doing that, the main lobe power increases while the sidelobe power is remaining nearly constant when observed over the azimuth cut. The achieved spatial taper does not reduce the overall sidelobe power but spreads it outside the elevation sector of interest. The width of the elevation sector where the sidelobes are reduced depends on the number of antenna elements in the z-domain. The number of elements for each row can be calculated by using (1). The general idea is that the number of antennas in the following row is increased

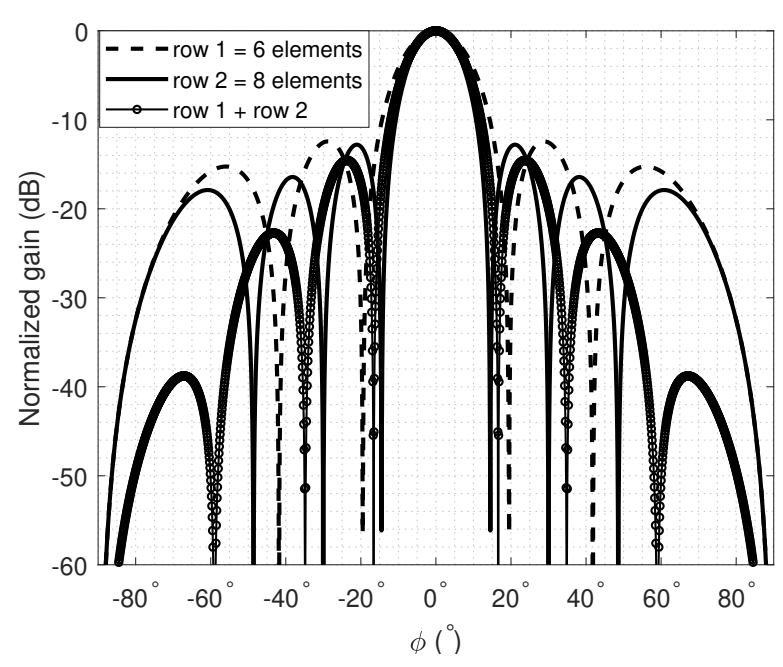

(a)

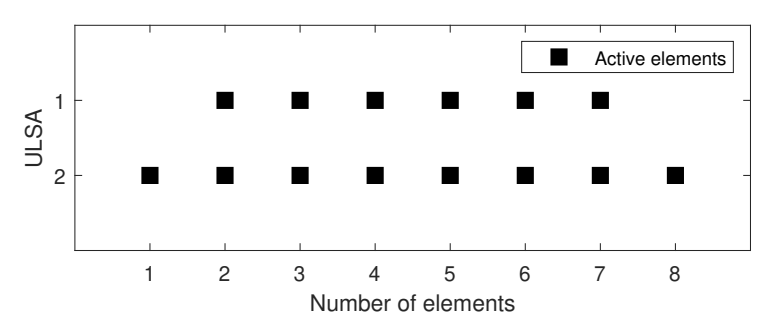

(b)

Fig. 3. (a) Simulated array factors of six- and eight-element rows and the combined array pattern, and (b) corresponding antenna configurations.

and selected such that it places a null in the location of the first sidelobe of the previous row. The sidelobe direction can be calculated as the center point between the two closest nulls next to the main beam by using (1). When observed over the azimuth plane in a zero-elevation angle, the stacking produces amplitude distribution, which gives more power for the center columns while the end of the rows produces less power. Hence, the vertical domain is used to taper the amplitude observed over the azimuth plane.

An example of the subarray stacking by using (1) with two rows is illustrated in Fig. 3. The first row has six elements, and the next row hence requires eight elements to place a null in the direction of the first sidelobe of the first row. The location of the sidelobes of each row is different.

1) Relaxation of the Null Direction for Sidelobe Reduction: If no rise in the SLL is allowed, the blind use of (1) may produce an unfeasible number of elements. However, sidelobe width is dependent on the number of elements. Therefore, the target null location is not needed to be precisely in the direction of the other sidelobe maxima and the tolerable error relies on the width of the null and the sidelobe. In order to take this into account and prevent an absurd number of antenna elements resulting from too accurate null placement, the null target can be relaxed for each row so that $\phi_{\text {null }} \in \phi_{\text {margin }}$, 


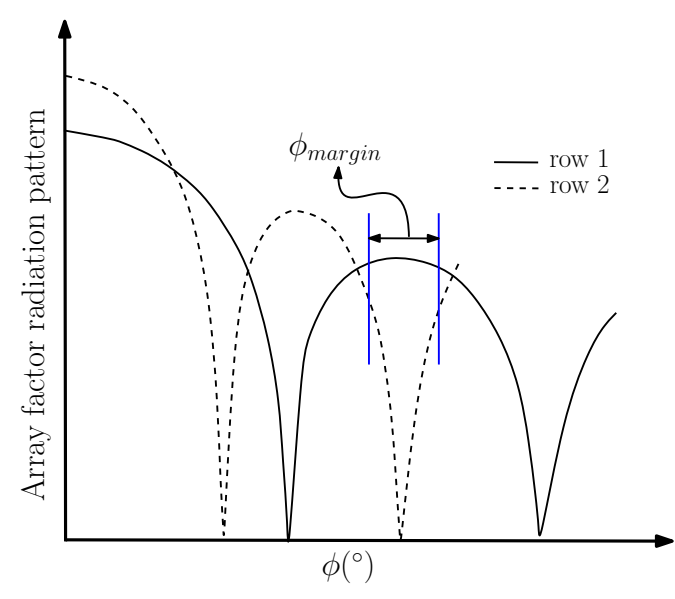

Fig. 4. Illustration of margin for relaxing the null direction for sidelobe reduction.

where $\phi_{\text {margin }}$ denotes the acceptable region of the null direction. In Fig. 4, the concept of the null margin for sidelobe reduction is shown. Rows have $N_{M}-2$ sidelobes and (1) can be used to calculate width of the sidelobes $\left(w_{s l}\right)$ between the nulls. The width of the sidelobe can be used as a starting point for determining the acceptable region of null directions for each row. Hence, the acceptable null margin for the $m+1$ antenna row can be written as

$$
\begin{aligned}
\phi_{\operatorname{margin}}(m+1) & \\
& =\left[\phi_{s l}(m)-\gamma w_{s l}(m), \phi_{s l}(m)+\gamma w_{s l}(m)\right]
\end{aligned}
$$

where $\phi_{s l}$ is the sidelobe maxima and $w_{s l}$ is the width of the first sidelobe of the row $m$. Equation (2) calculates the null margin for the row $m+1$ from the maximum sidelobe width and direction of the row $m$. For example, $\gamma=0.1$ indicates that the null direction must fall into $\pm 10 \%$ width of the sidelobe from the sidelobe maxima. Fig. 5 presents the simulation results of the total number of antenna elements of the 2D array as a function of the SLL with different threshold values. Larger null margin leads to a smaller number of antenna elements from row to row and vice versa, hence reducing the total number of elements. However, increasing the margin also decreases the sidelobe reduction performance. Hence, $\gamma$ can be used to compromise between the array size and SLL reduction performance. Simulation results also show that if more rows are stacked, SLL can be further reduced. The potential of the proposed technique is shown in Fig. 5. More than $30 \mathrm{~dB}$ of SLL for eight vertically stacked rows with 100 antenna elements can be achieved.

2) Procedure of Subarray Stacking for Sidelobe Reduction: The flow chart of the stacking procedure for sidelobe reduction with a relaxed null direction is shown in Fig. 6. The steps are further explained as follows.

1) The process is initialized by selecting the required number of rows $N_{\text {rows }}$, the maximum number of elements in a row $N_{\max }$, and the number of elements in the initial subarray $N_{1} . m$ denotes the row index. The selection of the input parameters depends on the physical constraints, i.e., how many rows and antennas we can allocate in total.

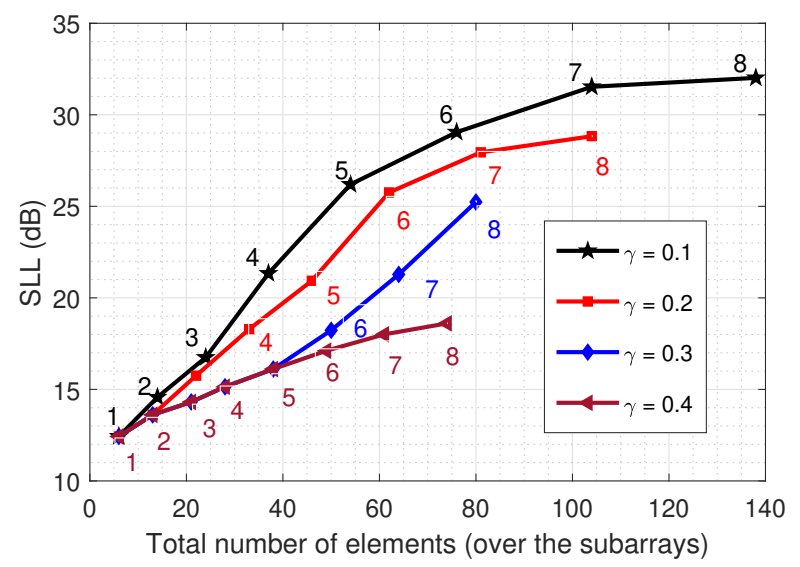

Fig. 5. Size of rows and relative SLL with different relaxation $(\gamma)$ for null directions. Simulations are made up to 8 rows of antennas.

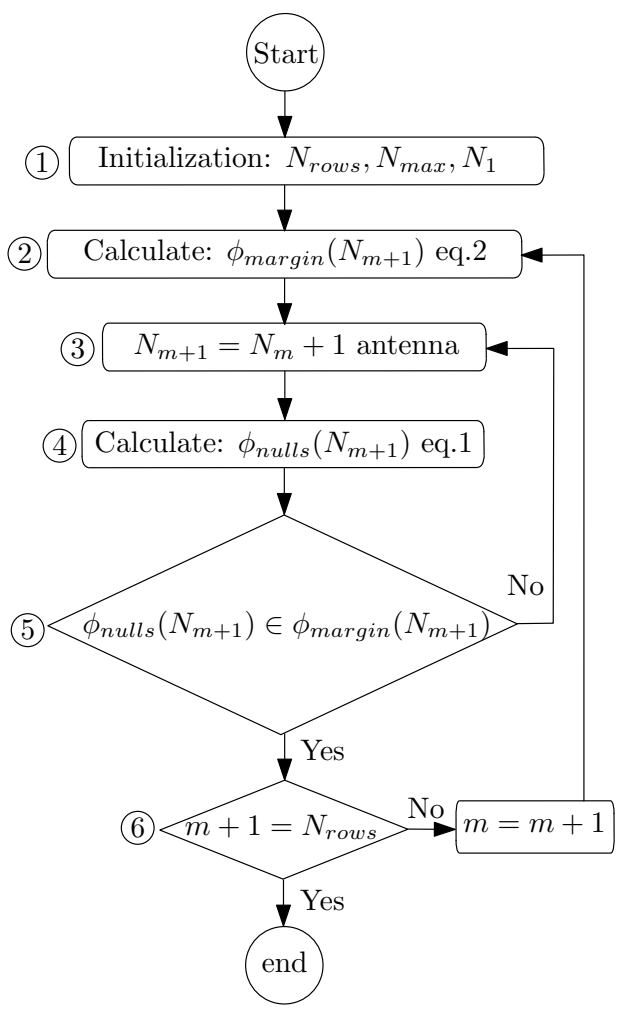

Fig. 6. Flow diagram of the subarray (row) stacking procedure for sidelobe reduction.

2) Acceptable range of the null $\phi_{\text {margin }}$ for the next row of antennas is calculated by using (2)

3) Subarray size is increased by one antenna $N_{m+1}=$ $N_{m}+1$

4) The null directions are calculated by using (1).

5) If any of the null directions falls into the acceptable range of nulls, that number of antennas is selected for the row $m+1$

6) Row index is checked. If the required number of subarrays $N_{\text {rows }}$ is completed, the procedure ends. Otherwise, it goes back to step (2), where the margin of the next subarray is calculated. 


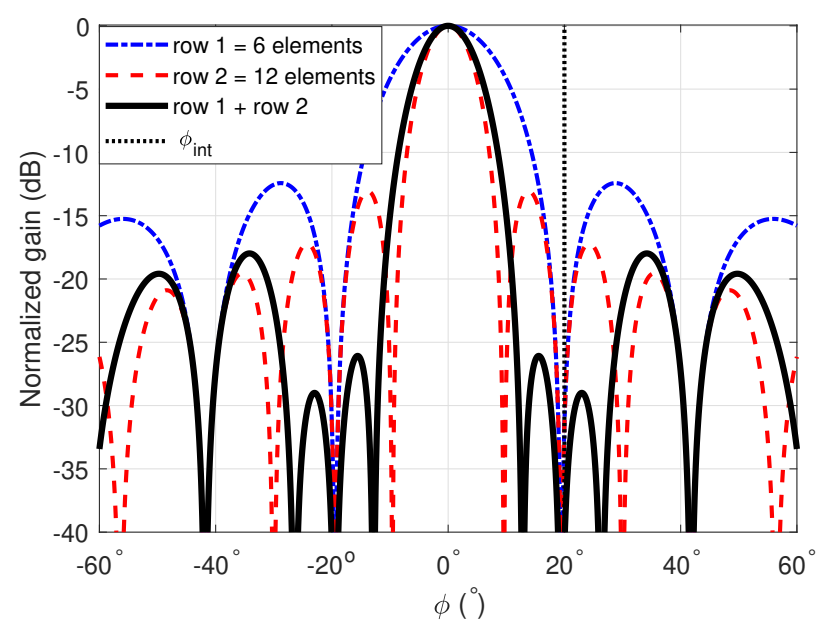

Fig. 7. Simulated array factor of 6- and 12-element rows and the combined array pattern.

\section{B. Subarray Stacking for Interference Nulling}

The radiated power can also be reduced to known directions of azimuth interference by stacking rows of different sizes in the elevation domain so that each row places a null to the interference direction $\phi_{\text {int }}$. Fig. 7 presents a simplified example of where 6- and 12-element ULSA's (rows) are stacked. Both rows produce a null in the direction of interference at 20 degrees in azimuth. The combined pattern of two rows reduces the interference in the chosen direction since the nulls of both subarrays fall approximately in the same direction. The obvious choice would be to stack rows of the same size to place nulls in the direction of known interference. However, if the number of elements in each row is varied as in the case of sidelobes reduction, besides the null in the desired direction, the sidelobes observed over the azimuth cut can be also simultaneously reduced. The resulted beam patterns over the azimuth cut are illustrated in Fig. 8 where the URA has higher sidelobes compared to the shape introduced in Fig. 7. The combined array forms a null in the desired direction and simultaneously decreases the level of sidelobes.

1) Relaxation of the Null Direction in Interference Nulling: Accurate nulling from stacked rows may produce an unfeasible number of elements in rows similarly, as in the case of sidelobe reduction. In order to avoid the too large number of antenna elements resulting from accurate null positioning, margin to reduce inter-beam interference $\left(\phi_{\operatorname{margin}(I B I)}\right)$ can be defined to ease the search for null directions in each row. A fixed margin for nulls from the stacked rows can be chosen as

$$
\phi_{\operatorname{margin}(I B I)}=\left[\phi_{i n t}-\phi_{e r r}, \phi_{i n t}+\phi_{e r r}\right],
$$

for the known direction of interference, where $\phi_{e r r}$ is the acceptable null direction error around the direction of interference illustrated in Fig. 9. A fixed margin was selected because the null width is not significantly varying when the number of elements is increasing. In the case of sidelobe reduction, the scenario was different as the sidelobe width had a relation to the number of antenna elements. Fig. 10 shows the margin

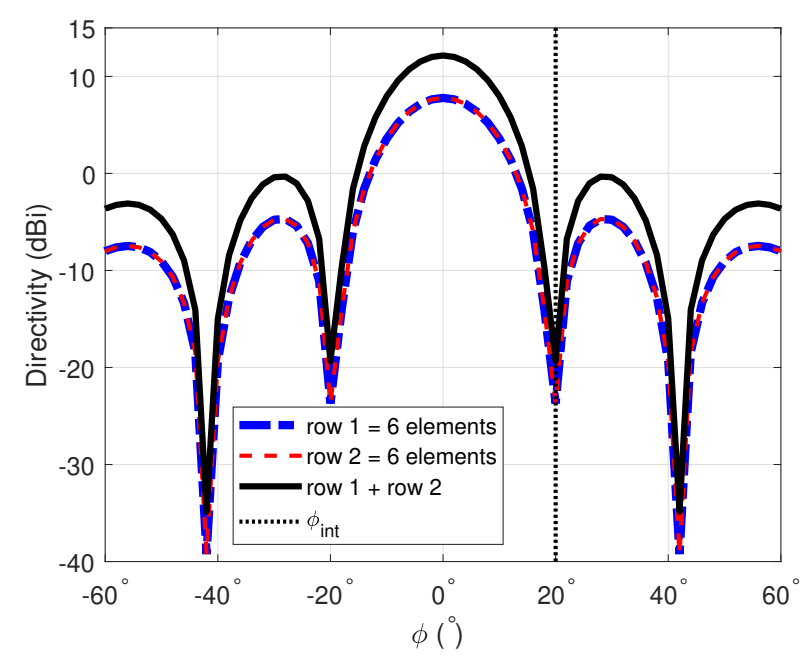

Fig. 8. Simulated array factor of two 6-element rows and the combined array pattern.

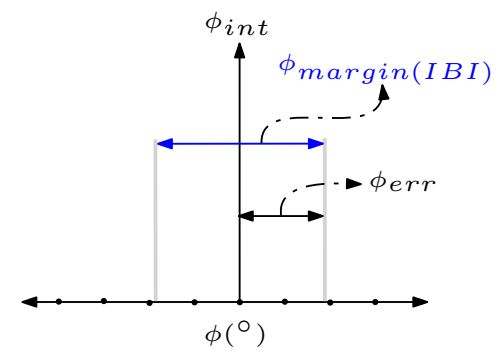

Fig. 9. Illustration of margin for nulls for known interferer.

for nulls and the number of elements of eight stacked rows. The elements are selected to reduce the interference in known direction (5-degree azimuth in this example) when the beam is targeted to zero degrees in azimuth. The small separation between the main beam and the interference direction was chosen in this example because if the number of elements is large, the interference is anyway reduced by the higher directivity (sidelobes becomes closer and closer to the main lobe). Hence, nulling interference has the best potential when the beams are located close to each other.

2) Procedure of Subarray Stacking for Interference Nulling: The procedure of subarray stacking for interference nulling is described in Fig. 11 and further explained as follows.

1) The process is initialized by selecting the required number of rows $N_{\text {rows }}$, the maximum number of elements in a row $N_{\max }$, and the number of elements in the initial subarray $N_{1} . m$ denotes the row index and $\phi_{s}$ the beam steering direction, and $\phi_{\text {int }}$ denotes the direction of the known interferer (desired null direction).

2) Acceptable range of the null direction $\phi_{\operatorname{margin}(I B I)}$ for the next row of antennas is calculated by using (3).

3) Subarray size is increased by one antenna $N_{m}=N_{m}+1$.

4) The null directions are calculated by using (1).

5) If any of the null directions fall into an acceptable range of nulls, that number of antennas is selected for the row $m+1$. 


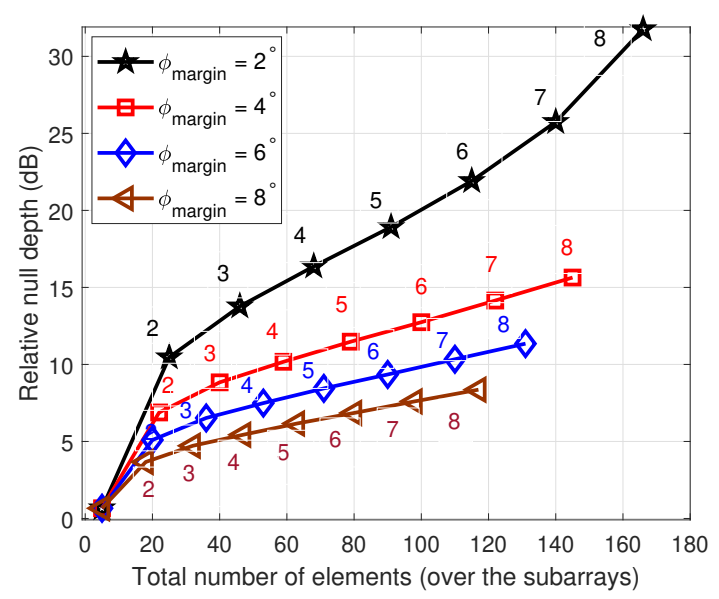

Fig. 10. Size of rows and relative null depth at 5 degrees offset with different null margins. Simulations are preformed up to 8 rows with the main beam steered to 0 degrees in azimuth.

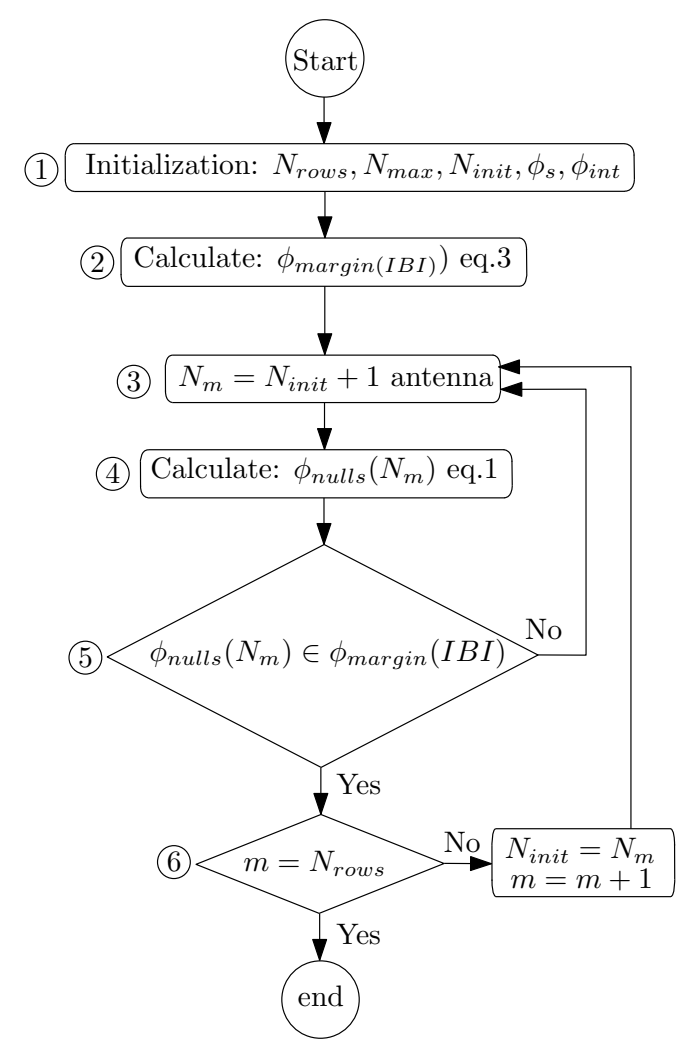

Fig. 11. Flow diagram of the subarray (row) stacking procedure for interference nulling.

6) Row index is checked. If the required number of subarrays $N_{\text {rows }}$ is achieved, the procedure ends. Otherwise, it goes back to step (3).

\section{Three-Dimensional Sidelobe Reduction and Nulling}

The antenna array geometries derived in the previous sections were designed for a static scenario without beam steering. Furthermore, stacking multiple elements in the vertical domain inevitably impacts the beam over the elevation angles.
Hence, the null depth and sidelobe level depend also on the elevation angle. Next, we evaluate the impact of both azimuth and elevation beam steering on the sidelobe reduction and nulling performances with the proposed array geometries.

When the rows are stacked with increasing length, both the sidelobe reduction and the nulling approaches produce triangular-like array geometries. For reference, we also simulated a scenario where half of the rows are chosen to have increasing length while the other half have decreasing length. This approach produces hexagonal-like array geometries that also provide spatial tapers in the elevation domain.

1) Three-Dimensional Sidelobe Reduction: The simulated beam patterns showing the sidelobe levels of three different antenna geometries are given in Fig. 12. The used rectangular, triangular, and hexagonal antenna configurations are illustrated in Figs. 12(a)-(c). Since the proposed approach spreads the sidelobes over both planes (azimuth and elevation), the simulations are performed over both domains. The normalized 3D beam patterns of rectangular, triangular and hexagonal geometries with beam steered to $\phi_{s}=0^{\circ}, \theta_{s}=0^{\circ}$ are shown in Figs. 12(d)-(f), respectively. For the rectangular geometry in Fig. 12(d), the sidelobes of each row co-locate in azimuth, producing $\sim 13 \mathrm{~dB}$ maximum sidelobe level that appears over the $\theta=0^{\circ}$ cut. For the triangular-like geometry, the sidelobes of some rows co-locate with the nulls of the other rows, producing reduced sidelobes over the $\theta=0^{\circ}$ cut. This is illustrated in the Fig. 12(e). The hexagonal shape enables one to focus even more power to the main lobe maintaining good sidelobe level also at larger elevation angles, as shown in Fig. 12(f). The hexagonal shape also gives better opportunities to control the sidelobe power simultaneously both in azimuth and elevation domain that can be critical in some link scenarios, e.g., antenna arrays placed on the ceiling.

To verify the impact of beam steering on sidelobe reduction for different antenna geometries, we apply azimuth beam steering while keeping the array geometries fixed. Figs. 12(g)(i) illustrate the beam pattern as a function of steering angle $\phi_{s}$ given on the $\mathrm{x}$-axis and different azimuth angles $\phi$ on the $\mathrm{y}$ axis. The normalized magnitude of the beam is illustrated with color-coding. It can be seen that azimuth cut is only slightly varying over \pm 50 degree of steering range. Based on Figs. 12(g)-(i) the sidelobe levels over azimuth domain are reduced for triangular and hexagonal shapes around the $\theta=0^{\circ}$ cut.

A similar analysis for elevation steering is shown in Figs. 12(j)-(l). The results are plotted with elevation steering angle $\theta_{s}$ on the $\mathrm{x}$-axis and elevation observation angle $\theta$ on the $y$-axis. The result corresponds to the azimuth beam steering indicating that the sidelobes follow both azimuth and elevation steering properly. Sidelobes of the hexagonal geometry are lower in the elevation plane compared to the rectangular or the proposed triangular-shaped arrays. One can choose between triangular or hexagonal geometries depending on the requirement of the spatial tapers in the elevation plane.

2) Three-Dimensional Nulling: As the sidelobes are spread over different elevation angles, the nulls are also expected to have a similar effect. The two example panel geometries, i.e., triangular and hexagonal, configured to produce a null, are given in Figs. 13(a) and (d). Here the target null direction is 


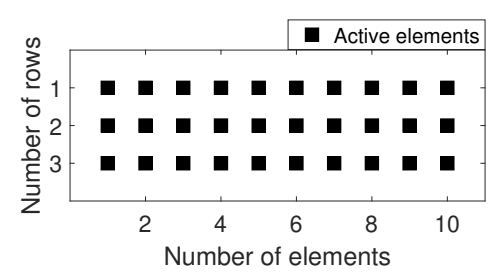

(a)

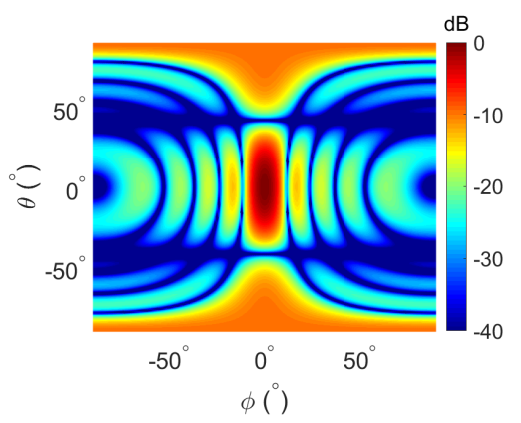

(d)

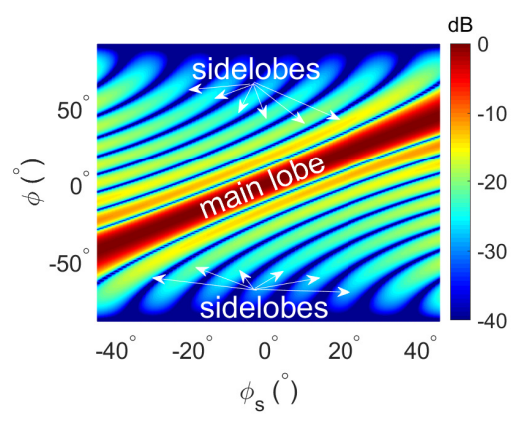

(g)

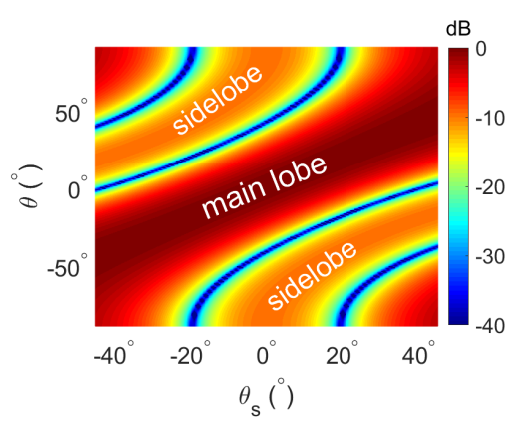

(j)

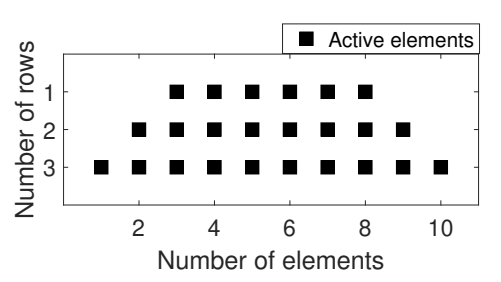

(b)

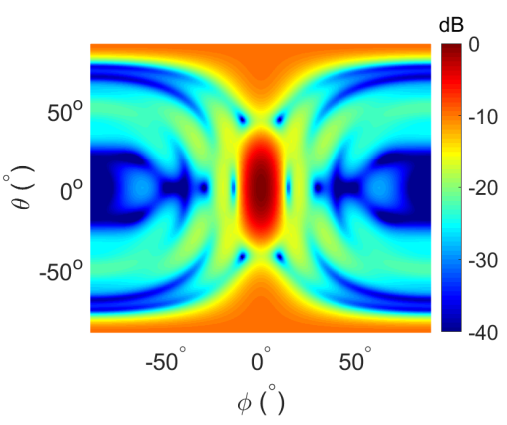

(e)

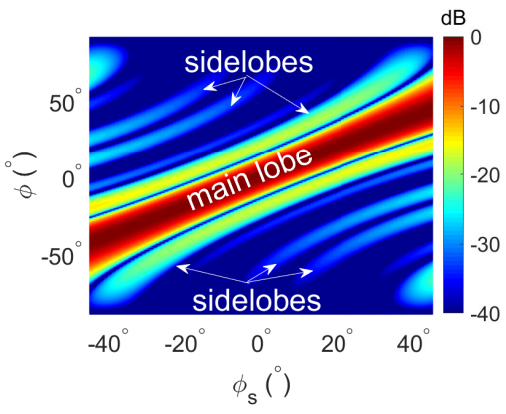

(h)

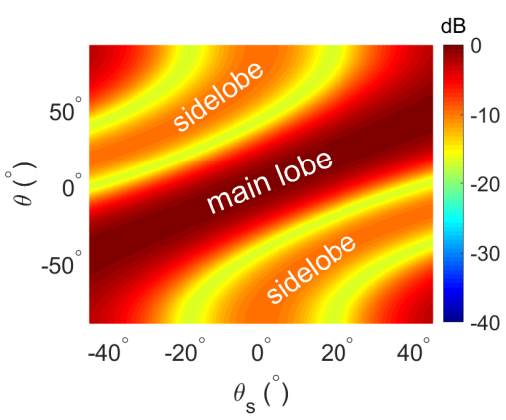

(k)

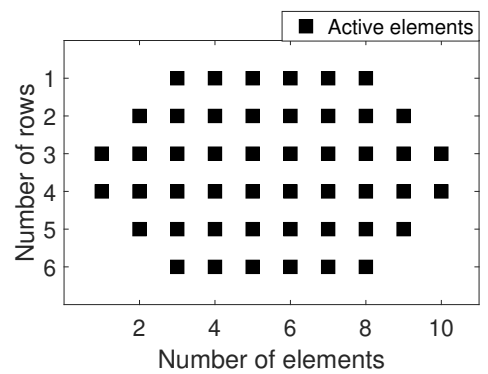

(c)

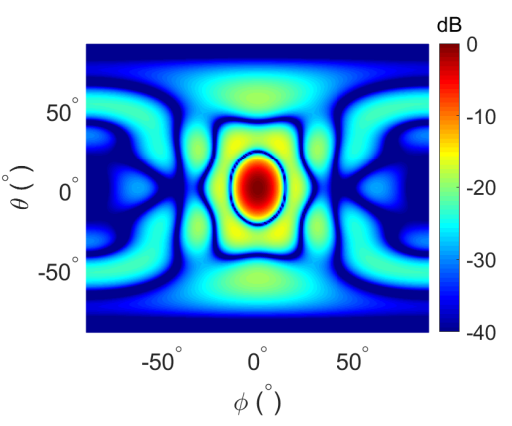

(f)

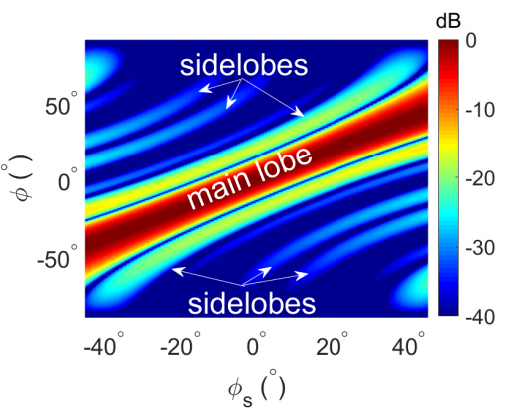

(i)

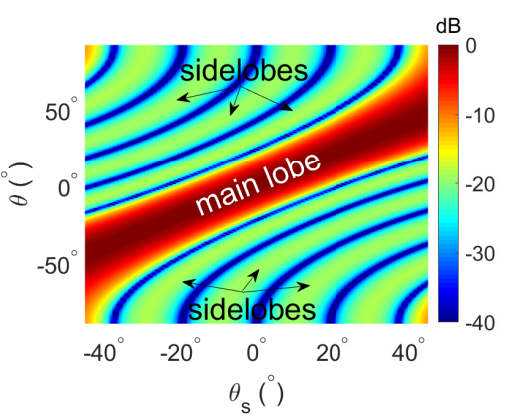

(1)

Fig. 12. Array geometry for (a) rectangular, (b) triangular, and (c) hexagonal configurations with the corresponding 3D beam patterns in (d), (e), and (f), respectively. Subfigures (g)-(i) are the corresponding $\theta=0^{\circ}$ cuts of the beams with respect to azimuth steering angle $\phi_{s}$ and (j)-(l) represents the $\phi=0^{\circ}$ cuts of the beams with respect to elevation steering angle $\theta_{s}$. 


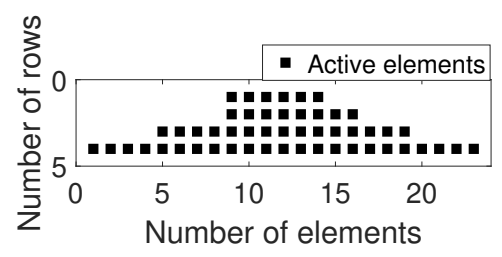

(a)

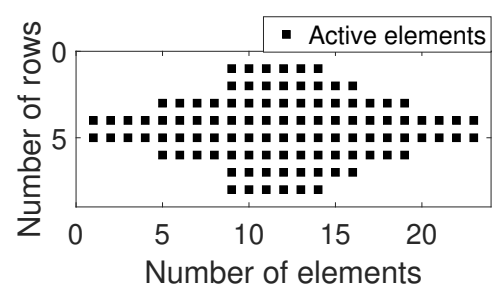

(d)

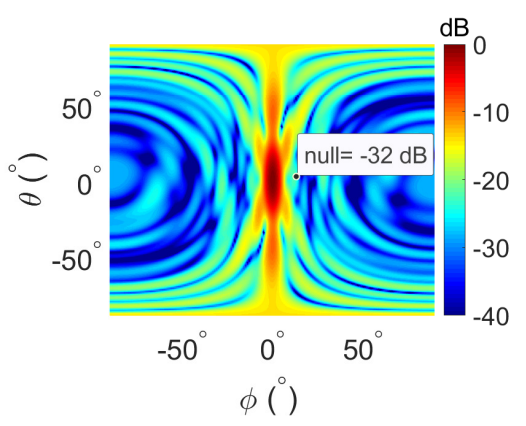

(b)

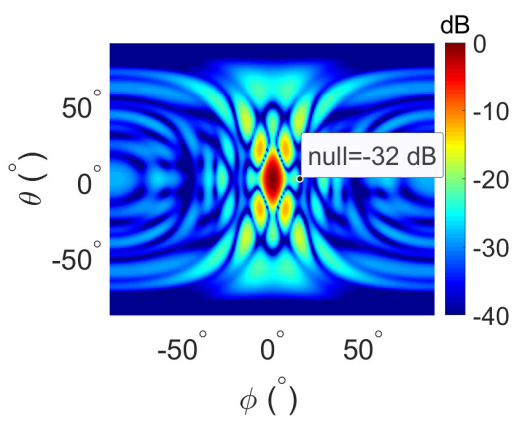

(e)

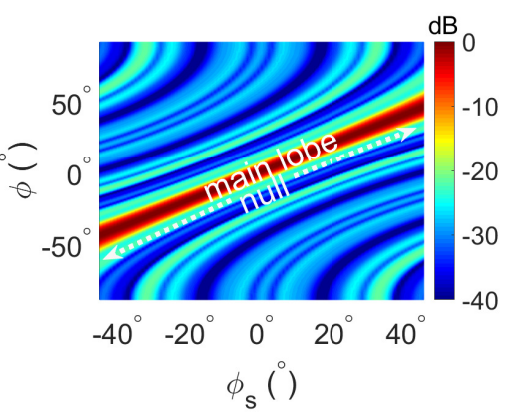

(c)

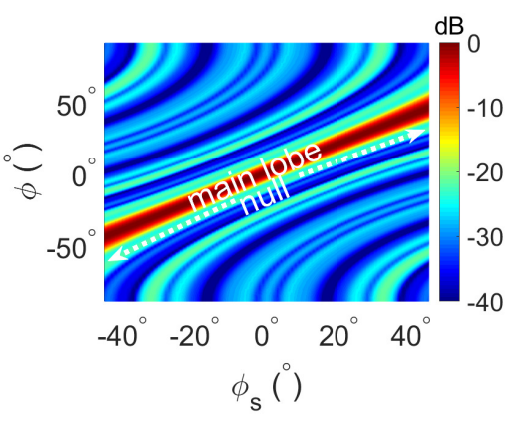

(f)

Fig. 13. Array geometries, 3D beam patterns, and $\theta=0^{\circ}$ cuts of the beams with respect to azimuth steering angle $\phi_{s}$ for (a)-(c) triangular and (d)-(f) hexagonal antenna configurations.

set to $\phi_{i n t}=15^{\circ}$ and $\theta_{i n t}=0^{\circ}$ when the beam is steered to boresight. The simulated $3 \mathrm{D}$ beam patterns are given in Figs. 13(b) and (e). The 3D beam patterns show that the null orientation and depth of the triangular and hexagonal geometries behave similarly, but the hexagonal geometry also provides spatial tapers for sidelobes in the elevation plane.

Next, we simulated the impact of the azimuth beam steering to the beam patterns of the proposed geometries. For simplicity, the geometries are kept fixed for each steering angle. Hence, the null direction is expected to move along with the scanning angle, similarly to the sidelobes moved in the previous section. The $\theta=0^{\circ}$ cuts of the beams for azimuth steering angle $\phi_{s}$ are shown in Figs. 13(c) and (f). As expected, with small scanning angles, the nulls move with the main lobe. However, with larger scanning angles, the null direction starts to slightly deviate from the intended angle.

\section{Two-beam Simulation Example of Interference Nulling}

When the main beam is steered, the nulls around it also change their positions. The null equation includes the impact of phase progression $\beta_{m y}$ to the location of nulls when the beam is steered. Given that the proposed technique also works when the beam is steered to the desired direction and nulling interference to the other direction is maintained by stacking appropriately sized subarrays (rows). A multibeam case is simulated where a beam is directed to one user, while nulls from the stacked rows are placed in the direction of the other user. Fig. 14 (a) shows the simulation of two beams where the beam of one user is at -5 degrees azimuth, and the other beam for the second user is at +10 degrees azimuth. In both beams, four rows are stacked, as shown in Fig. 14 (b). For both beams, the number of elements in rows changes, which moves the sidelobe direction of the rows, resulting in convenient power distribution in the SL-region as well as null interference in the direction of the other user.

\section{Experimental Validation with 28 GHz Phased ARRAY TRANSCEIVER}

We use a 64-element antenna array [44] designed originally for mobile backhaul application to demonstrate the proposed interference reduction method in practice. The array is divided into $2 \times 2$ groups specified as unit cells shown in Fig. 15(a). Each unit cell has four linearly polarized patch antenna elements that are slanted -45 degrees from the vertical. The spacing is half a wavelength $(=5.4 \mathrm{~mm})$ at $28 \mathrm{GHz}$ between the individual antenna elements. The simulated radiation pattern of a single unit cell is shown in Fig. 15(b). 16 unit cells are placed in an $8 \times 2$ array formation with $\lambda$ spacing between the cells. Hence, 64 individual antenna elements fed by 16 transceiver (TRx) ports are used. The simplified block diagram of the transceiver is shown in Fig. 16. The TRx switches of each antenna branch are used to turn branches on and off to configure the 2D shape of the antenna array. The transceiver array operates in the receive mode in the measurements. More details of the phased array TRx implementation is available in [45], [46].

The block diagram of the measurement setup located in an anechoic chamber is presented in Fig. 17. Each of the 16 TRx branches has individual TRx switches, low noise amplifiers (LNAs), PAs, and 5-bit phase shifters. Keysight M8190A arbitrary waveform generator (ARB) and E8257B 


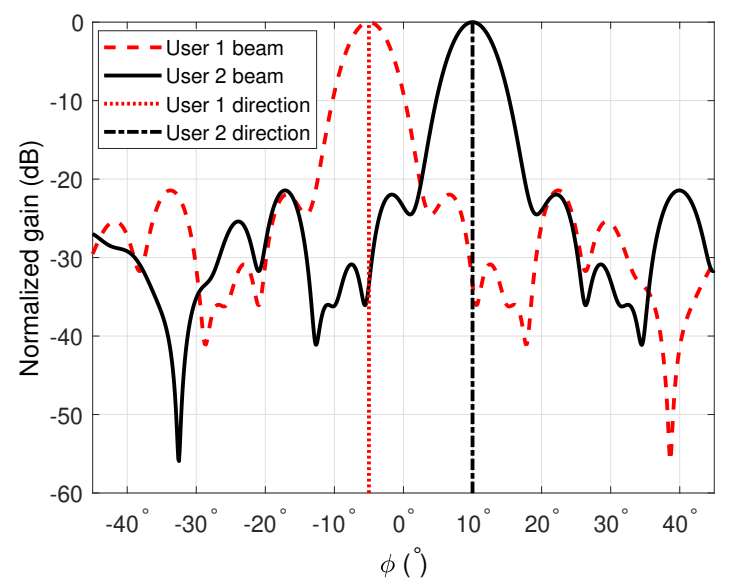

(a)

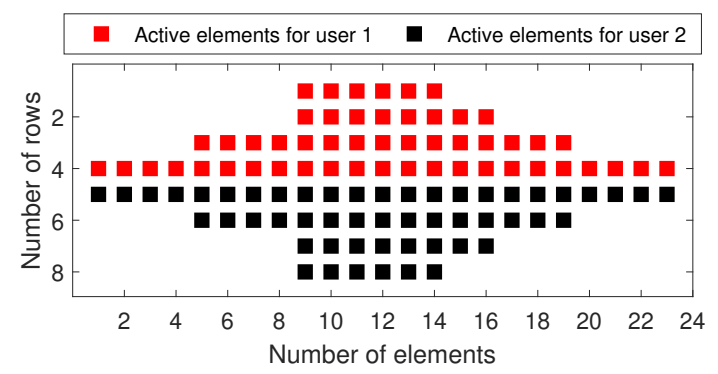

(b)

Fig. 14. Two-beam scenario (a) and interference reduction (b) for panel selection.

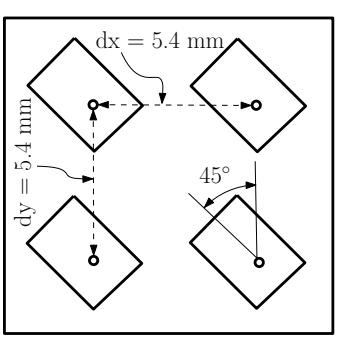

(a)

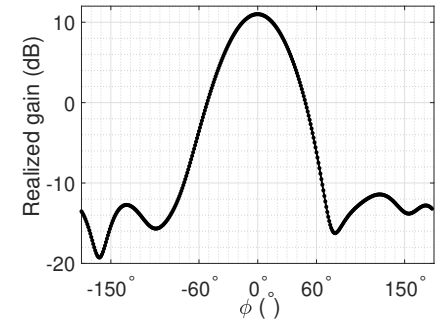

(b)
Fig. 15. (a) $2 \times 2$ unit cell antenna array with a single feed and (b) horizontal cut of the simulated radiation pattern of the unit cell.

PSG signal generator are used to generate a $100 \mathrm{MHz}$ wide 16quadrature amplitude modulation (QAM) cyclic-prefix orthogonal frequency division multiplexing (CP-OFDM) waveform at $28 \mathrm{GHz}$ center frequency according to the 3GPP 5G NR standard. In the transmission, the signal is amplified by using CA2630-141 external amplifier and fed to A-info LB-28-15 standard gain horn antenna located at 2 meters distance in the far-field of the device under test (DUT). The phased array receiver output is fed to the UXA signal analyzer, which is used to measure the channel power of the signal over the $100 \mathrm{MHz}$ band. The DUT is placed on a rotating table to measure the radiation pattern over the azimuth quarter plane. The measurements are conducted at $28 \mathrm{GHz}$ center frequency with 1-degree angle resolution over $\phi \in\left[-45^{\circ}, 45^{\circ}\right]$. For

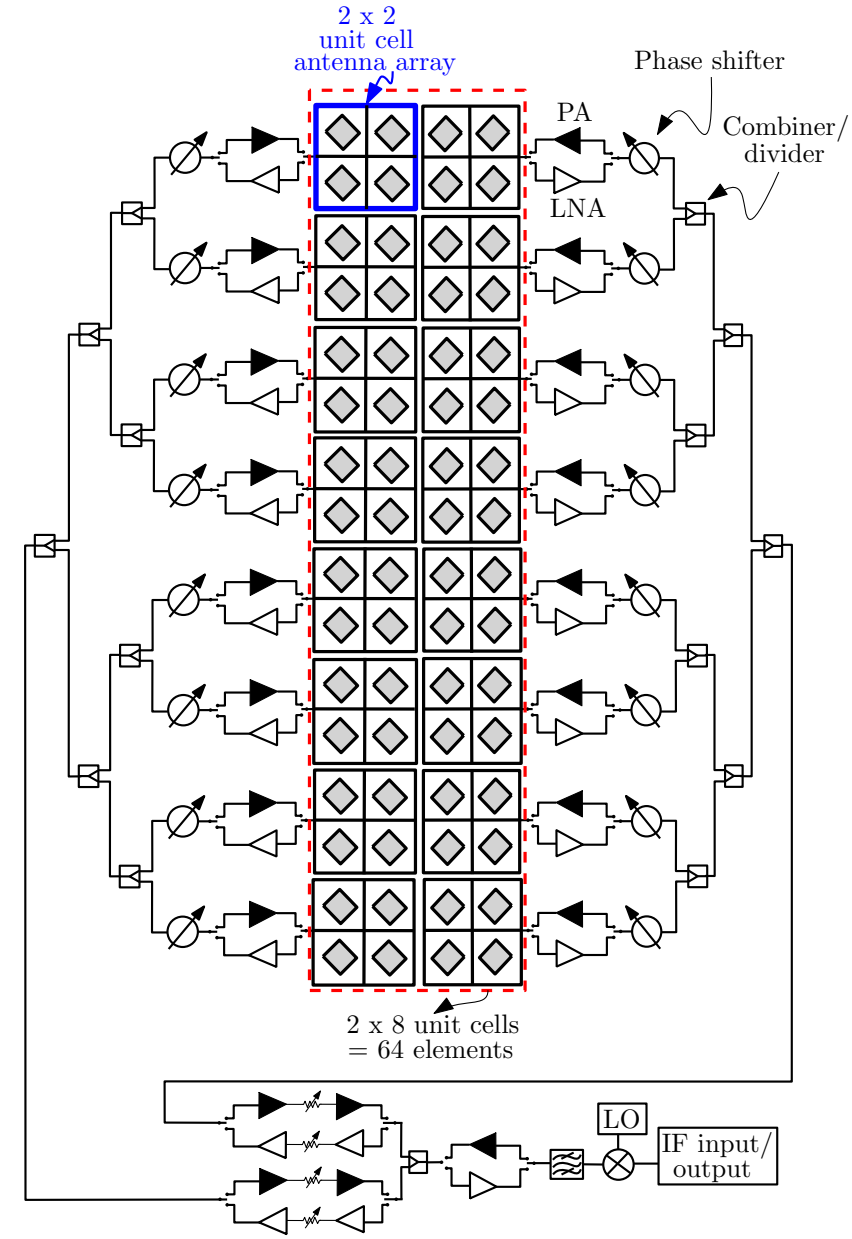

Fig. 16. Simplified block diagram of the $28 \mathrm{GHz}$ phased array transceiver under test.

simplicity, the phase shifters are calibrated to the 0-degree azimuth angle in the example. However, it should be noted that the measured interference configuration also works when the beam is steered in azimuth domain. A photograph of the measurement setup in the chamber is shown in Fig. 18. Due to the limitation of only 16 ports in the transceiver array (with two independent rows), one configuration for the sidelobe reduction and one configuration for the known interference reduction is demonstrated.

\section{A. Measurement Results for Sidelobe Reduction}

For sidelobe reduction, the demonstrated configuration has four $2 \times 2$ antenna unit cells in the first row and six in the second row shown in Fig. 19(a). In total, 40 antenna elements are active. For the comparison, we also measured a rectangular configuration with 40 elements in 10x4 formation as shown in 19(b). The measurement results are presented in Fig. 20 for sidelobe reduction.

The radiation patterns of both measured configurations are not symmetric due to the antenna implementation and nonequal gains of the receiver branches [45], [46]. The symmetricity of the pattern and hence the results can be improved by calibrating the element gains to be equal. However, due to the lack of the per-antenna amplitude control, that was not 


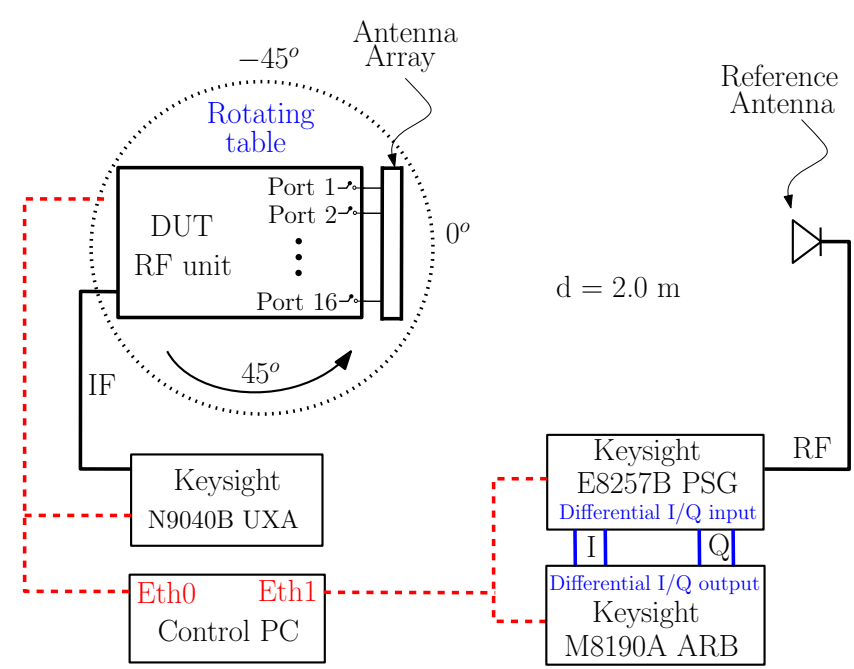

Fig. 17. Block diagram of the over-the-air measurement setup with $100 \mathrm{MHz}$ wide $5 \mathrm{G}$ waveform at $28 \mathrm{GHz}$.

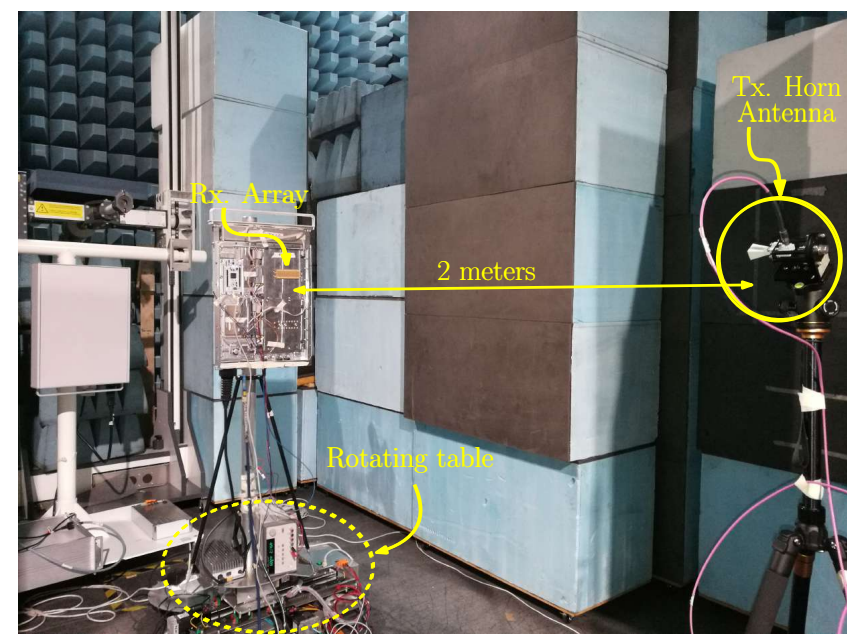

Fig. 18. Photograph of the measurement arrangement in the anechoic chamber.

possible in our platform. In the measurements, the receiver gain and the gain of the horn antenna are compensated from the results. The first sidelobe of the first row and the null of the second row are aligned by the proposed configuration thereby reducing the total sidelobes. The SLL results of the maximum right and the left sidelobe levels are collected to the Table I. The measurements show 2.5 and $2.6 \mathrm{~dB}$ sidelobe reduction with only two rows of unit cells. The additional loss in the reduction performance compared to theory is also partly caused by the wideband measurement with the modulated signal, which decreases the notch depth when the power is integrated over the whole $100 \mathrm{MHz}$ signal band. Measurement results indicate that the proposed sidelobe reduction technique works in practice. With a larger number of rows, low sidelobe levels can be achieved to reduce the interference even further.

\section{B. Measurement Results for Interference Nulling}

For the known direction of the interference reduction, the demonstrated configuration has four $2 \times 2$ antenna unit cells in
TABLE I

Measured SLls of Proposed (Prop) and Rectangular (Rect) ANTENNA CONFIGURATIONS

\begin{tabular}{|c|c|c|c|}
\hline Meas. Rect (right) & $10.9 \mathrm{~dB}$ & Meas. Prop (right) & $13.4 \mathrm{~dB}$ \\
\hline Meas. Rect (left) & $12.8 \mathrm{~dB}$ & Meas. Prop (left) & $15.4 \mathrm{~dB}$ \\
\hline
\end{tabular}

Active elements $\diamond$ Inactive elements

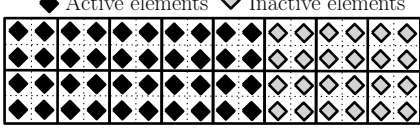

(a)

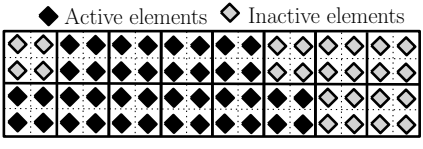

(b)
Fig. 19. 64-element, 16-chain antenna panel with (a) the proposed configuration for SLL reduction and (b) rectangular $4 \times 10$.

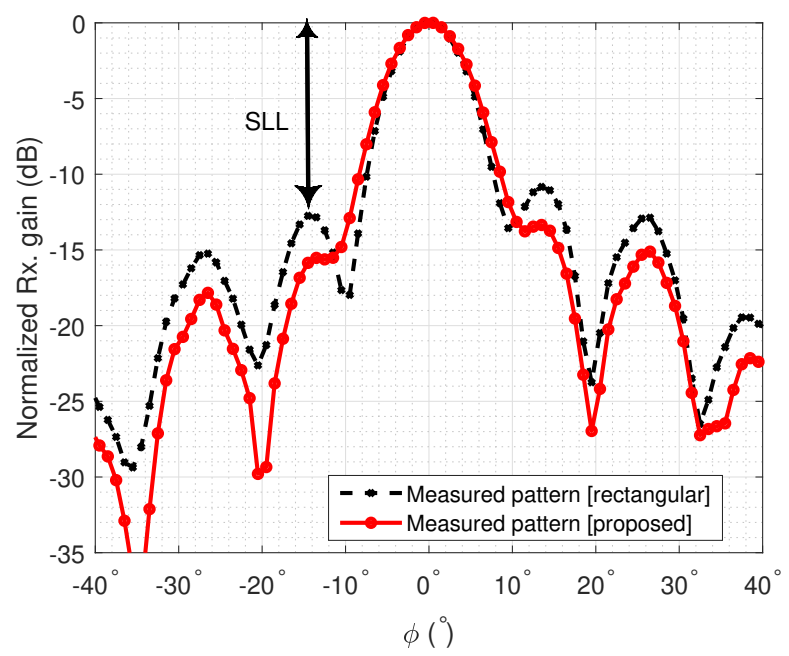

Fig. 20. Horizontal cuts of the measured radiation patterns of the rectangular and the proposed triangular antenna configuration.

the first row and eight in the second row shown in Fig. 21(a). In total, 48 antenna elements are active. We also measured a rectangular configuration of $12 \times 4$ array having 48 elements for comparison, as shown in Fig. 21(b). Measurement result for reducing the interference in the known direction is presented in Fig. 22. The measured patterns are normalized to zero to demonstrate the null depth with the proposed configuration. Rows of the same size have the sidelobe in the direction of interference. The proposed configuration places a null in the known direction of interference. Rows of different length also have different locations of sidelobes, which do not add up power in the direction of sidelobes and therefore attain reduced relative sidelobes along with placing null in the interference direction.

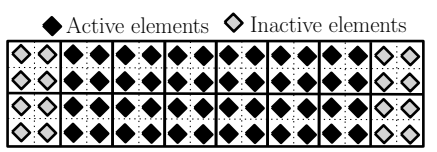

(a)

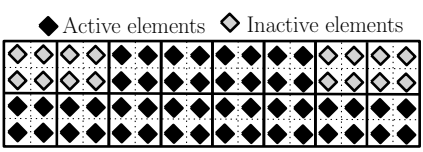

(b)
Fig. 21. 64-element, 16-chain antenna panel with (a) the proposed configuration for nulling, and (b) rectangular $4 \times 12$ array for reference. 


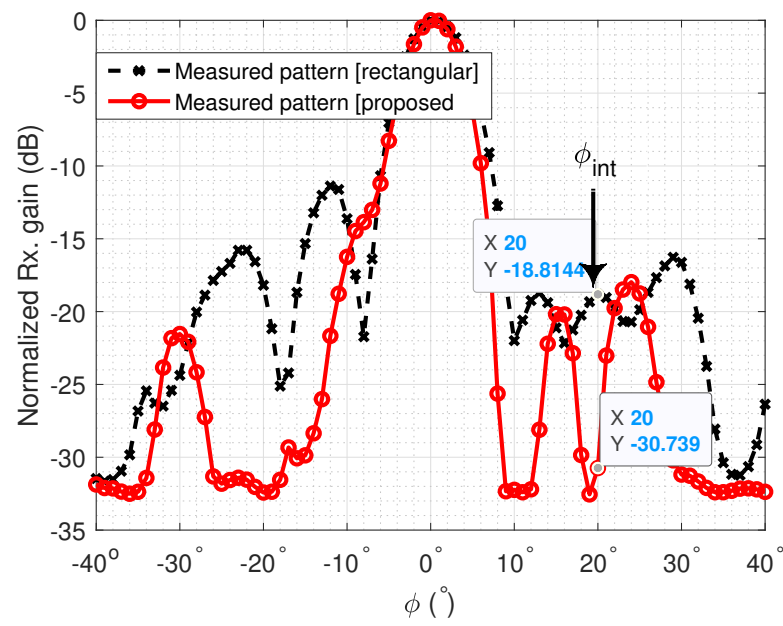

Fig. 22. Horizontal cuts of the measured radiation patterns of the rectangular and proposed triangular subarrays.

TABLE II

Measured Null of Proposed (Prop) and Rectangular (Rect) ANTENNA CONFIGURATIONS

\begin{tabular}{|c|c|c|c|}
\hline Meas. Rect (right) & $18.8 \mathrm{~dB}$ & Meas. Prop (right) & $30.7 \mathrm{~dB}$ \\
\hline Meas. Rect (left) & $18.1 \mathrm{~dB}$ & Meas. Prop (left) & $32.4 \mathrm{~dB}$ \\
\hline
\end{tabular}

\section{CONCLUSION}

In $\mathrm{mmW}$ arrays, different antenna geometries and element configurations produce alternative ways of performing beamforming compared to the classical rectangular shape. A method of interference reduction was proposed by stacking multiple rows in the vertical domain to decrease interference over the azimuth plane. The method was utilized for single-direction null placement and sidelobe reduction. For sidelobe reduction, the number of antennas in each row was varied to align the nulls and maxima of the rows to increase the main lobe gain while the sidelobe power remained constant, hence improving the sidelobe level. The specific interference direction was nulled by selecting the number of elements to produce a null in a specific direction and stacking arrays of different lengths to reduce the sidelobes simultaneously. It was shown that the null direction is not required to be exact to achieve decent interference performance, and hence the method was relaxed by introducing a margin relative to the sidelobe width of each antenna row. The proposed interference reduction technique was demonstrated by using a 64-element phasedarray transceiver operating at $28 \mathrm{GHz}$ center frequency. Overthe-air measurements were carried out in an anechoic chamber by using $100 \mathrm{MHz}$ wide CP-OFDM waveform following the 3GPP 5G NR standard. The proposed configurations of the subarray stacking method were compared against a standard rectangular array with the same total number of antenna elements. The measurement results show $2.5 \mathrm{~dB}$ of sidelobe reduction with only two rows of antennas compared to the reference rectangular antenna configuration. In the case of nulling of a single direction, more than $10 \mathrm{~dB}$ of interference reduction compared to the rectangular was achieved with only two rows. In both cases, the performance potential of the proposed method improves for larger arrays.

It is possible to use numerous beamforming methods to form nulls and reduce the sidelobes, but they often require amplitude control per antenna, which reduces the maximum achievable radiated power. However, the proposed method used the maximum available power per antenna to reduce sidelobe or nullify interference in any particular direction. In practice, the technique can be used in phased arrays having a large number of antenna elements. The antenna geometries can be varied during the operation, and hence the array size and configuration are not required to be fixed for a specific scenario. The proposed technique gives an alternative way of simplifying the interference reduction and achieving better beam separation in multi-user and multi-beam systems. The technique can be implemented in practice in transceivers using various techniques, for example, by using the TDD switch of each branch to turn elements on and off during the operation.

\section{REFERENCES}

[1] M. Xiao et al., "Millimeter Wave Communications for Future Mobile Networks," IEEE J. Sel. Areas Commun., vol. 35, no. 9, pp. 1909-1935, 2017.

[2] E. G. Larsson et al., "Massive MIMO for Next Generation Wireless Systems," IEEE Commun. Mag., vol. 52, no. 2, pp. 186-195, 2014.

[3] S. A. Busari et al., "Millimeter-Wave Massive MIMO Communication for Future Wireless Systems: A Survey," IEEE Commun. Surveys Tuts., vol. 20 , no. 2 , pp. 836-869, 2018.

[4] S. Sun et al., "Propagation Models and Performance Evaluation for 5G Millimeter-Wave Bands," IEEE Trans. Veh. Technol., vol. 67, no. 9, pp. 8422-8439, 2018.

[5] Z. Wang et al., "An Overlapped Subarray Structure in Hybrid Millimeter-Wave Multi-User MIMO System," in Proc. IEEE Global Commun. Conf., 2018, pp. 1-6.

[6] E. Björnson et al., "Optimal Multiuser Transmit Beamforming: A Difficult Problem With a Simple Solution Structure [Lecture Notes]," IEEE Signal Process. Mag., vol. 31, no. 4, pp. 142-148, 2014.

[7] C. A. Balanis, Antenna theory: analysis and design, 4th ed. Wiley, 2015.

[8] T. Tuovinen et al., "Analyzing 5G RF System Performance and Relation to Link Budget for Directive MIMO," IEEE Trans. Antennas Propag., vol. 65, no. 12, pp. 6636-6645, 2017.

[9] A. Wiesel et al., "Zero-Forcing Precoding and Generalized Inverses," IEEE Trans. Signal Process., vol. 56, no. 9, pp. 4409-4418, 2008.

[10] J. Cong et al., "Hybrid MMSE Beamforming for Multiuser MillimeterWave Communication Systems," IEEE Commun. Lett., vol. 22, no. 11, pp. 2390-2393, 2018.

[11] K. Huang et al., "Spatial Interference Cancelation for Mobile Ad Hoc Networks: Imperfect CSI," in Proc. IEEE 42nd Asilomar Conf. Signals, Syst.,Comput., 2008, pp. 131-135.

[12] T. Yoo et al., "Multi-Antenna Downlink Channels with Limited Feedback and User Selection," IEEE J. Sel. Areas Commun., vol. 25, no. 7, pp. 1478-1491, 2007.

[13] J. Fan and M. Wu, "Antenna Selection in Switched Phased Array Architecture for Secure Millimeter Wave Communication," in Proc. IEEE 17th Int. Conf. Commun. Tech. (ICCT), 2017, pp. 718-723.

[14] W. Dong et al., "Auxiliary Elements Selection for Planar Array Sidelobe Cancellation Based on Genetic Algorithm," in Proc. Eur. Conf. Antennas Prop. (EUCAP), 2018, pp. 1-5.

[15] X. Wang and E. Aboutanios, "Adaptive Beampattern Synthesis With Antenna Selection by Iterative Shrinkage Continuation Method," in Proc. IEEE 21st Eur. Signal Process. Conf. (EUSIPCO), 2013, pp. 1-5.

[16] Q. Lijun et al., "Antenna Subarray Division and Adaptive Beamforming," in Proc. IEEE Radar Conf., 1996, pp. 484-487.

[17] Ching-Tai Lin and Hung Ly, "Sidelobe Reduction Through Subarray Overlapping for Wideband Arrays," in Proc. IEEE Radar Conf., 2001, pp. 228-233.

[18] M. C. Viganò et al., "Sparse Planar Array Synthesis Technique for Satellite Applications," in Proc. IEEE Int. Symp. Antennas and Propag. Soc., 2010, pp. 1-4. 
[19] M. Y. Javed et al., "Sidelobe Reduction by Subarray Stacking for Uniformly Excited mmW Phased Arrays," in Proc. IEEE 30th Annu. Int. Symp. Pers., Indoor Mobile Radio Commun. (PIMRC), 2019, pp. $1-5$.

[20] C. L. Dolph, "A Current Distribution for Broadside Arrays Which Optimizes the Relationship Between Beam Width and Side-Lobe Level," Proc. IRE, vol. 34, no. 6, pp. 335-348, 1946.

[21] D. Rhodes, "On the Taylor Distribution," IEEE Trans. Antennas Propag., vol. 20, no. 2, pp. 143-145, 1972.

[22] O. S. Ginting, Chairunnisa, and A. Munir, "Side Lobe Level Suppression for L-band Array Antenna Using Binomial Power Distribution," in Proc. 3rd Int. Conf. Wireless Telematics (ICWT), 2017, pp. 7-10.

[23] A. K. Aboul-Seoud et al., "A Sidelobe Level Reduction (SLL) for Planar Array Antennas," in Proc. Nat. Radio Sci. Conf., 2009, pp. 1-8.

[24] M. Y. Javed, N. Tervo, and A. Pärssinen, "Inter-Beam Interference Reduction in Hybrid mmW Beamforming Transceivers," in Proc. IEEE 29th Annu. Int. Symp. Pers., Indoor Mobile Radio Commun. (PIMRC), 2018, pp. 220-224.

[25] R. V. Gatti, M. Dionigi, and R. Sorrentino, "Computation of Gain, Noise Figure, and Third-Order Intercept of Active Array Antennas," IEEE Trans. Antennas Propag., vol. 52, no. 11, pp. 3139-3143, 2004.

[26] A. Ishimaru, "Theory of Unequally-Spaced Arrays," IRE Trans. Antennas Propag., vol. 10, no. 6, pp. 691-702, 1962.

[27] R. L. Haupt, "Adaptively Thinned Arrays," IEEE Trans. Antennas Propag., vol. 63, no. 4, pp. 1626-1632, 2015.

[28] L. Feng, G. Cui, X. Yu, and L. Kong, "Beampattern Synthesis via the Constrained Subarray Layout Optimization," IEEE Trans. Antennas Propag., 2020

[29] Y. Lo and S. Lee, "A Study of Space-Tapered Arrays," IEEE Trans. Antennas Propag., vol. 14, no. 1, pp. 22-30, 1966.

[30] S. Edelberg and A. Oliner, "Mutual Coupling Effects in Large Antenna Arrays: Part 1-Slot Arrays," IRE Trans. Antennas Propag., vol. 8, no. 3, pp. 286-297, 1960.

[31] D. G. Leeper, "Isophoric Arrays - Massively Thinned Phased Arrays With Well-Controlled Sidelobes," IEEE Trans. Antennas Propag., vol. 47 , no. 12 , pp. $1825-1835,1999$.

[32] B. Sadhu et al., "A 28-GHz 32-Element TRX Phased-Array IC With Concurrent Dual-Polarized Operation and Orthogonal Phase and Gain Control for 5G Communications," IEEE J. Solid-State Circuits, vol. 52, no. 12, pp. 3373-3391, 2017.

[33] S. Shahramian et al., "A Fully Integrated 384-Element, 16-Tile, $W$ Band Phased Array With Self-Alignment and Self-Test," IEEE J. SolidState Circuits, vol. 54, no. 9, pp. 2419-2434, 2019.

[34] T. Sowlati et al., "A 60-GHz 144-Element Phased-Array Transceiver for Backhaul Application," IEEE J. Solid-State Circuits, vol. 53, no. 12, pp. 3640-3659, 2018.

[35] I. E. Lager et al., "Interleaved Array Antennas for FMCW Radar Applications," IEEE Trans. Antennas Propag., vol. 57, no. 8, pp. $2486-$ 2490, 2009.

[36] R. L. Haupt, "Interleaved Thinned Linear Arrays," IEEE Trans. Antennas Propag., vol. 53, no. 9, pp. 2858-2864, 2005.

[37] T. Isernia, M. D'Urso, and O. M. Bucci, "A Simple Idea for an Effective Sub-Arraying of Large Planar Sources," IEEE Antennas Wireless Propag. Lett., vol. 8, pp. 169-172, 2009.

[38] V. Pierro et al., "Radiation Properties of Planar Antenna Arrays Based on Certain Categories of Aperiodic Tilings," IEEE Trans. Antennas Propag., vol. 53, no. 2, pp. 635-644, 2005.

[39] P. Angeletti and G. Toso, "Aperiodic Arrays for Space Applications: A Combined Amplitude/Density Synthesis Approach," in Proc. Eur. Conf. Antennas Prop. (EUCAP), 2009, pp. 2026-2030.

[40] W. Zhai, V. Miraftab, and M. Repeta, "Randomly Tiled Rectangular SubArrays for Side Lobe and Grating Lobe Reduction in mm-Wave Limited Scanning Phased Array," in Global Symp. Millimeter Waves (GSMM), 2016, pp. 1-4.

[41] W. Zhai, M. Repeta, D. Wessel, and W. Tong, "mm-Wave Large-Scale Phased Array Based on Randomly Tiled Rectangular Sub-Arrays for 5G Communications," in IEEE MTT-S Int. Microw. Symp. (IMS)., 2017, pp. 1895-1898.

[42] R. Akbar et al., "A Wideband IF Receiver Module for Flexibly Scalable mmWave Beamforming Combining and Interference Cancellation," in IEEE Eur. Solid State Circuits Conf. (ESSCIRC), 2019, pp. 213-216.

[43] P. Rocca and R. L. Haupt, "Dynamic Thinning Strategy for Adaptive Nulling in Planar Antenna Arrays," in Proc. IEEE Phased Array Symp., 2010, pp. 995-997.

[44] M. Sonkki et al., "Linearly Polarized 64-element Antenna Array for mm-Wave Mobile Backhaul Application," in Proc. Eur. Conf. Antennas Prop. (EUCAP), 2018, pp. 1-5.
[45] M. E. Leinonen et al., " $28 \mathrm{GHz}$ Wireless Backhaul Transceiver Characterization and Radio Link Budget," ETRI J., vol. 40, no. 1, pp. 89-100, 2018.

[46] O. Kursu et al., "Design and Measurement of a $5 \mathrm{G} \mathrm{mmW}$ Mobile Backhaul Transceiver at $28 \mathrm{GHz}$," EURASIP J. Wireless Commun. Netw., vol. 2018, no. 1, p. 201, 2018.

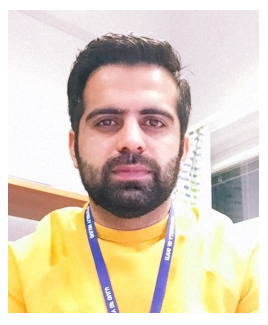

Muhammad Yasir Javed (Graduate Student Member, IEEE) received the B.Eng. degree in telecommunication from National University of Modern Languages (NUMLs), Islamabad, Pakistan, in 2013. He received his M.Sc. degree in wireless communication engineering from University of Oulu, Oulu, Finland, in 2017, where he is currently pursuing the $\mathrm{Ph} . \mathrm{D}$. degree at the Centre for Wireless Communications (CWC). His current research interests include millimeter-wave, phased array, multibeam RF architectures and interference reduction techniques.

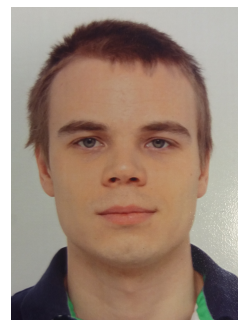

Nuutti Tervo (Member, IEEE) received the B.Sc. (Tech.) and M.Sc. (Tech.) degrees in electrical engineering from the University of Oulu, Oulu, Finland, in 2014, where he is currently pursuing the Ph.D. degree at the Centre for Wireless Communications (CWC). His Ph.D. dissertation research focuses on nonlinearity and linearization of millimeter-wave beamforming transceivers. He has a strong background in different fields of wireless communications, including radio frequency (RF), radio channel modeling, signal processing, and system-level analysis. Around those topics, he has already authored or coauthored more than 35 international journal and conference papers.

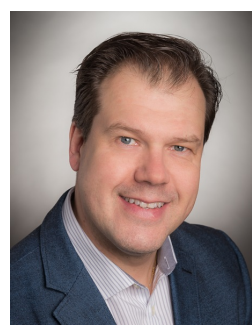

Marko E. Leinonen (Member, IEEE) received the M.Sc. and Licentiate in Technology degrees in electrical engineering from the University of Oulu, Oulu,Finland, in 1996 and 2002, respectively. From 1994 to 2012, he was with Nokia Mobile Phones, Oulu, working in various positions with radio engineering and technology management. From 2006 to 2007, he was a Senior Engineering Manager in Bengaluru, India. From 2012 to 2016, he was a Master Developer with Ericsson, Oulu. Since 2017, he has been with the Centre for Wireless Communications, University of Oulu, where he is currently the Research Manager. He holds 40 granted international patent families concentrating on radio engineering. His research interests include wireless radio systems and quality topics in radio engineering. 


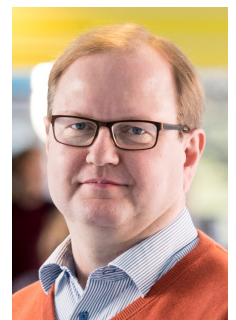

Aarno Pärssinen (Senior Member, IEEE) received the M.Sc., Licentiate in Technology, and Doctor of Science degrees in electrical engineering from the Helsinki University of Technology, Finland, in 1995, 1997, and 2000, respectively.

From 1994 to 2000, he was with the Electronic 1139 Circuit Design Laboratory, Helsinki University of Technology, Espoo, Finland, working on direct conversion receivers and subsampling mixers for wireless communications. In 1996, he was a Research Visitor with the University of California at Santa 1144 Barbara, Santa Barbara, CA, USA. From 2000 to 2011, he was with the Nokia Research Center, Helsinki, Finland. From 2009 to 2011, he was a member of the Nokia CEO Technology Council. From 2011 to 2013, he was with Renesas Mobile Corporation, Helsinki, working as a Distinguished Researcher and an RF Research Manager. From October 2013 to September 2014, he was an Associate Technical Director with Broadcom Corporation, Helsinki. Since September 2014, he has been with the Centre for Wireless Communications, University of Oulu, Oulu, Finland, where he is currently a Professor. He is also leading devices and circuits research area in 6G flagship program financed by the Academy of Finland. His research interests include wireless systems and transceiver architectures for wireless communications with special emphasis on the radio frequency (RF) and analog integrated circuit and system design. He has authored or coauthored one book, two book chapters, more than 150 international journal and conference papers, and holds several patents. He is also one of the original contributors to Bluetooth lowenergy extension, now called BT LE.

Dr. Pärssinen served as a member for the Technical Program Committee of the International Solid-State Circuits Conference from 2007 to 2017, where he was the Chair of the European Regional Committee from 2012 to 2013 and the Chair of the Wireless Sub-Committee from 2014 to 2017. He has been serving as a Solid-State Circuits Society Representative for the IEEE 5G Initiative from 2015 to 2019. 